\title{
Identifying preoperative language tracts and predicting postoperative functional recovery using HARDI q-ball fiber tractography in patients with gliomas
}

\author{
*Eduardo Caverzasi, MD, ${ }^{1,2}$ Shawn L. Hervey-Jumper, MD, ${ }^{3}$ Kesshi M. Jordan, BS, ${ }^{4}$ \\ Iryna V. Lobach, PhD, ${ }^{5}$ Jing Li, MD, ${ }^{3}$ Valentina Panara, MD, ${ }^{6}$ Caroline A. Racine, PhD, ${ }^{3}$ \\ Vanitha Sankaranarayanan, MS, ${ }^{7}$ Bagrat Amirbekian, BS, ${ }^{1,4}$ Nico Papinutto, PhD, ${ }^{1}$ \\ Mitchel S. Berger, MD, ${ }^{3}$ and Roland G. Henry, PhD ${ }^{1,4,7}$
}

Departments of ${ }^{1}$ Neurology, ${ }^{3}$ Neurological Surgery, ${ }^{5}$ Epidemiology and Biostatistics, and ${ }^{7}$ Radiology and Biomedical Imaging, University of California, San Francisco, California; '2Department of Medical Imaging, St. Michael's Hospital, Toronto, Ontario, Canada; ${ }^{4}$ Graduate Program in Bioengineering, University of California, Berkeley/University of California, San Francisco, California; and ${ }^{6}$ Institute of Advanced Biomedical Technologies, University G. D’Annunzio, Chieti, Italy

OBJECTIVE Diffusion MRI has uniquely enabled in vivo delineation of white matter tracts, which has been applied to the segmentation of eloquent pathways for intraoperative mapping. The last decade has also seen the development from earlier diffusion tensor models to higher-order models, which take advantage of high angular resolution diffusion-weighted imaging (HARDI) techniques. However, these advanced methods have not been widely implemented for routine preoperative and intraoperative mapping.

The authors report on the application of residual bootstrap q-ball fiber tracking for routine mapping of potentially functional language pathways, the development of a system for rating tract injury to evaluate the impact on clinically assessed language function, and initial results predicting long-term language deficits following glioma resection.

METHODS The authors have developed methods for the segmentation of 8 putative language pathways including dorsal phonological pathways and ventral semantic streams using residual bootstrap q-ball fiber tracking. Furthermore, they have implemented clinically feasible preoperative acquisition and processing of HARDI data to delineate these pathways for neurosurgical application. They have also developed a rating scale based on the altered fiber tract density to estimate the degree of pathway injury, applying these ratings to a subset of 35 patients with pre- and postoperative fiber tracking. The relationships between specific pathways and clinical language deficits were assessed to determine which pathways are predictive of long-term language deficits following surgery.

RESULTS This tracking methodology has been routinely implemented for preoperative mapping in patients with brain gliomas who have undergone awake brain tumor resection at the University of California, San Francisco (more than 300 patients to date). In this particular study the authors investigated the white matter structure status and language correlation in a subcohort of 35 subjects both pre- and postsurgery. The rating scales developed for fiber pathway damage were found to be highly reproducible and provided significant correlations with language performance. Preservation of the left arcuate fasciculus (AF) and the temporoparietal component of the superior longitudinal fasciculus (SLF-tp) was consistent in all patients without language deficits $(p<0.001)$ at the long-term follow-up. Furthermore, in patients with short-term language deficits, the AF and/or SLF-tp were affected, and damage to these 2 pathways was predictive of a long-term language deficit $(p=0.005)$.

CONCLUSIONS The authors demonstrated the successful application of q-ball tracking in presurgical planning for language pathways in brain tumor patients and in assessing white matter tract integrity postoperatively to predict long-term

ABBREVIATIONS AF = arcuate fasciculus; AFTD = altered fiber tractography density; $C D$ = clinical deficit; CRT set = clinically relevant tract set; DTI = diffusion tensor imaging; FA = fractional anisotropy; GTR = gross-total resection; HARDI = high angular resolution diffusion-weighted imaging; HGG = high-grade glioma; IFOF = inferior frontooccipital fasciculus; ILF = inferior longitudinal fasciculus; IR-SPGR = inversion-recovery spoiled gradient echo; ISM = intraoperative stimulation mapping; LGG = low-grade glioma; MdLF = middle longitudinal fasciculus; MNI = Montreal Neurological Institute; MST set = minimally sufficient tract set; ODF = orientation distribution function; ROI = region of interest; SLF = superior longitudinal fasciculus; SLF-tp = temporoparietal component of SLF; SLF-II\&III = SLF components II and III; UF = uncinate fasciculus. SUBMITTED September 25, 2014. ACCEPTED June 8, 2015.

INCLUDE WHEN CITING Published online December 11, 2015; 10.3171/2015.6.JNS142203.

* Drs. Berger and Henry share senior authorship. 


\begin{abstract}
language dysfunction. These initial results predicting long-term language deficits following tumor resection indicate that
\end{abstract} postoperative injury to dorsal language pathways may be prognostic for long-term clinical language deficits.

Study results suggest the importance of dorsal stream tract preservation to reduce language deficits in patients undergoing glioma resection, as well as the potential prognostic value of assessing postoperative injury to dorsal language pathways to predict long-term clinical language deficits.

http://thejns.org/doi/abs/10.3171/2015.6.JNS142203

KEY WORDS brain tumor; white matter; language; tractography; q-ball; presurgical planning; oncology

$\mathrm{I}$ $\mathrm{N}$ the last decade, the concept of human brain functionality has undergone dramatic changes. It has shifted from a rigid brain organization consisting of highly specialized regions toward a more dynamic view of human brain processes,,$^{12}$ characterized by interactions of large-scale networks underlying complex brain functions, such as language. Extensive resection in functional brain regions is possible without irreversible neurological deficits. This is probably due to a reshaping of brain functionality, occurring particularly in slow-growing tumors. Though the central nervous system shows high plastic potential, ${ }^{23,29}$ subcortical white matter plasticity is limited; thus damage to these bundles may result in more severe clinical deficits than will cortical lesions. ${ }^{12,13}$ White matter is critical to brain reorganization. ${ }^{12,13}$ It represents the substrate on which functional plasticity develops, first by recruiting local latent networks within the peritumoral region and later by recruiting from distant homolateral or contralateral regions. ${ }^{13}$

The goal of surgery for intrinsic brain tumors is maximal safe resection ${ }^{8,26}$ with preservation of quality of life and neurological function. Complex functions, such as language, are subserved by a multicomponent organization consisting of ventral and dorsal pathways. ${ }^{11,16}$ To reduce postoperative morbidity, increasing effort has been made to spare key white matter pathways underlying large-scale networks. To accomplish this, the precise characterization of white matter organization is crucial during presurgical planning. The model generated by fiber tractography with diffusion tensor imaging (DTI), which represents the standard procedure for presurgery fiber tracking in patients with brain tumors, has contributed greatly to our knowledge of language fiber pathways. However, this model suffers from several shortcomings in resolving the extent, origin, and cortical termination of white matter pathways. Recently, tractography algorithms based on diffusion models using high angular resolution diffusion-weighted imaging (HARDI) have been developed, ${ }^{4,31}$ along with complementary probabilistic methods. ${ }^{4}$ In particular, qball algorithms have been shown to improve the resolution of white matter fiber tractography in regions with crossing fiber populations..$^{3,5,14,21}$ The application of updated tracking methods to presurgery planning for language tracking in brain tumor patients enables the exploration of white matter pathways that could not be accurately reconstructed using older DTI methods.

The presurgery program delineating functional white matter pathways that we implemented 15 years ago ${ }^{3,14}$ has benefited from significant technological developments. This program started with delineation of the corticospinal tract using DTI fiber tracking ${ }^{4,5,21}$ and then evolved to include probabilistic q-ball fiber tracking methods ${ }^{4}$ and visual pathways. Routine delineation of language-related pathways, however, is not well established, and we do not know of any other centers that are using advanced fiber tracking methods, for example, residual bootstrap q-ball fiber tracking. Furthermore, there are scarce published data on the relationships between these pathways and clinically assessed language function. Important decisions regarding which pathways are vital to language processing and the short- or long-term impact on language function are not well informed. To pursue the overarching goal of answering these questions, our presurgery mapping program has been applying q-ball tracking to localize language pathways belonging to the ventral or dorsal pathways since January 2012. Individual patient data were processed and uploaded to the intraoperative neuronavigation system to guide direct subcortical stimulation for the identification of language-related structures.

We reviewed the imaging and perioperative clinical data of patients who had undergone awake craniotomy for language mapping with subcortical stimulation for tumor removal in the left hemisphere, as well as the tracking results obtained from q-ball tractography pre- and postsurgery. Our aims were to 1) evaluate the feasibility and reproducibility of our residual bootstrap probabilistic q-ball fiber tracking in presurgery planning for patients with brain tumor, 2) correlate fiber tracking results with language function presurgery, 3) explore the feasibility of postsurgery fiber tracking reconstruction, and 4) correlate postsurgery fiber tracking results with long-term language function to study its predictive power for recovery if affected by resection. Results of initial studies are reported herein.

\section{Methods}

\section{Subjects}

Research was performed in compliance with the Code of Ethics of the World Medical Association (Declaration of Helsinki) and the standards established by our institution. The Committee on Human Research at the University of California, San Francisco, approved the study protocol. Written informed consent was obtained from all study participants.

Since January 2012, when our presurgical languagemapping program was implemented for clinical use, more than 300 patients have undergone this protocol. Their diffusion data are acquired the day prior to surgery, and qball tracking is performed to localize language pathways belonging to either ventral or dorsal streams. These pathway reconstructions are uploaded to the neuronavigation 
system used by the surgeon during intraoperative stimulation mapping (ISM) (Fig. 1).

We selected from this cohort of patients a subcohort that had undergone awake surgery for tumor removal (WHO Grade II or higher) with subcortical stimulation between January 2012 and September 2013 (78 cases). Inclusion criteria were as follows: left-hemisphere surgery, no prior resections, and availability of pre- and postsurgery MRI acquisitions (within 1-2 days of surgical treatment) with HARDI data.

\section{Clinical Evaluation}

Age, sex, clinical history, symptoms, and neurological status were recorded. To identify both temporary and permanent language deficits, all patients underwent neurological assessment with language testing prior to surgery (baseline), at discharge (within 3 days of surgery), and at the long-term follow-up (>3 months postsurgery). Language testing was performed to evaluate language production, repetition, and comprehension. The following tests were performed: spontaneous speech, reading, object naming, comprehension, and counting. Language function evaluation was performed in accordance with prior published reports. ${ }^{15,25,26}$ Baseline language testing was performed 24-48 hours prior to surgery and included counting from 1 to 50, naming (64-object panel), reading, and spelling. Two independent clinicians conducted postoperative language testing: an attending neurosurgeon, a neurosurgical resident, and/or an attending neurooncologist. Differences between the results of the 2 examinations were adjudicated by accepting the results showing greater impairment. Outpatient clinical examinations were performed at 1 week, 4-6 weeks, and 3-6 months after surgery. Language laterality was assigned using navigated transcranial magnetic stimulation and magnetoencephalographic imaging, ${ }^{28}$ and handedness was noted as well. Surgery was performed using cortical and subcortical ISM for language and motor functions. Neuronavigation (Brainlab) was used in all cases to define tumor location, design the bone flap, and determine tumor boundaries after opening the dura mater. Direct cortical and subcortical stimulation mapping was performed using an Ojemann stimulator $(60-\mathrm{Hz}, 1-\mathrm{msec}-$ duration biphasic square-wave pulses, Integra, Inc.). Electrocorticography was used to determine the threshold at which afterdischarge potentials occurred. Mapping was performed using a stimulation intensity $1 \mathrm{~mA}$ below the threshold that produced afterdischarges (ranging from 3 to $5.5 \mathrm{~mA}$ ). Subcortical stimulation during tumor resection used the same stimulation parameters. ${ }^{9,18}$ Both cortical and subcortical language testing was performed in all cases. Testing included picture naming, reading, and counting (to identify speech arrest). According to our previously established protocol, speech arrest was tested by asking patients to count and was defined as the absence of speech without pharyngeal or mouth motor activation. A 1-cm or less margin was preserved around functional cortical language sites; however, the tumor resection was continued subcortically until any stimulation-induced language dysfunction occurred..$^{24,26}$ The identification of cortical and subcortical language and motor sites was considered the limit of the resection.
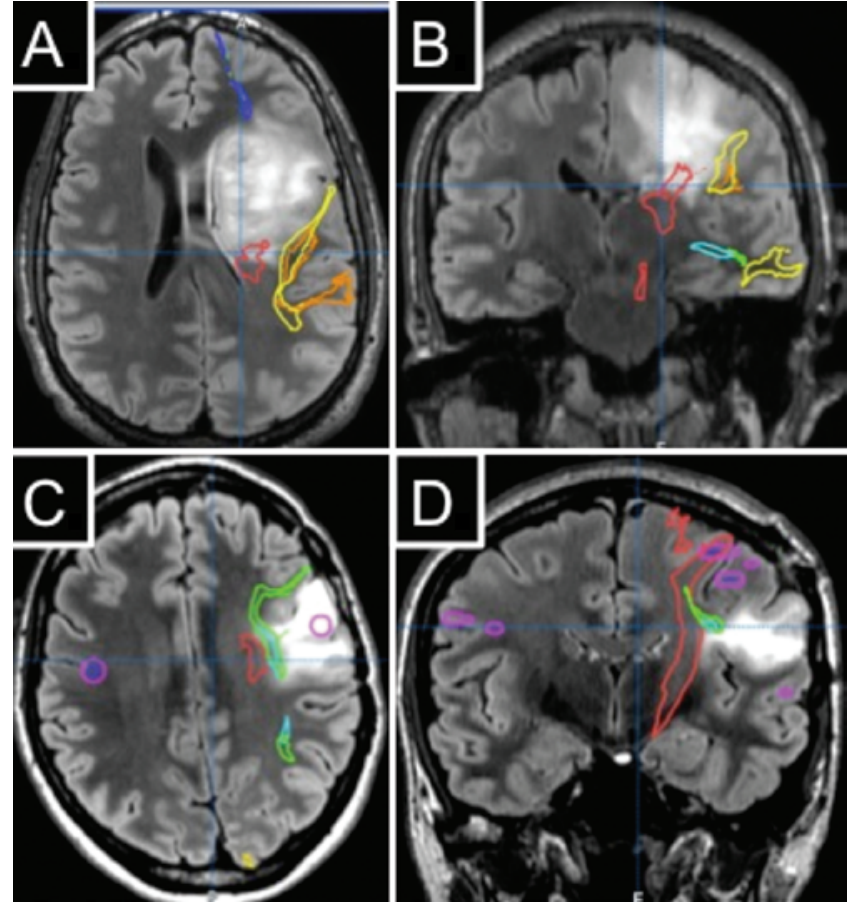

FIG. 1. Intraoperative neuronavigation MRI with residual bootstrap q-ball tracking results. Case 31. Axial (A) and coronal (B) FLAIR sequences obtained in a patient with a WHO Grade III anaplastic oligodendroglioma displacing the corticospinal tract (red), superior longitudinal fasciculus (SLF; orange), and arcuate fasciculus (AF; yellow) posteriorly and laterally. Note that the patient had a language deficit presurgery, which was still present postsurgery. Case 36. Axial (C) and coronal (D) FLAIR sequences obtained in a patient with a WHO Grade II oligoastrocytoma growing lateral to the corticospinal fibers (red) and below language pathways (SLF-II\&III, green; AF, teal). Note that the patient did not show any language deficit pre- or postsurgery.

\section{Magnetic Resonance Imaging Acquisition}

Subjects were scanned pre- and postsurgery with a 3-T General Electric Medical Systems scanner (Discovery MR750). Both HARDI (TR $6425 \mathrm{msec}$, TE $80 \mathrm{msec}$, axial slices 50 , isotropic voxel $2.2 \mathrm{~mm}^{3}$, b value $2000 \mathrm{~s} / \mathrm{mm}^{2}$, diffusion gradients 55, minimally diffusion-weighted image 1) and FLAIR data sets were acquired pre- and postsurgery. Three-dimensional high-resolution T1-weighted inversion-recovery spoiled gradient-echo (IR-SPGR; TR 7 msec, TE 2 msec, TI 400 msec, axial slices 180, thickness $1 \mathrm{~mm}$, in-plane resolution $0.94 \times 0.94 \mathrm{~mm}^{2}$ ) sequences were acquired only before surgery.

\section{Volumetric Analyses}

For each subject, the tumor was segmented manually across all slices with region-of-interest (ROI) analysis to compute pre- and postoperative volumes. Volumetric measures were calculated by segmenting low-grade hyperintense regions on axial FLAIR images and high-grade tumor regions of enhancement on postcontrast T1-weighted images. The percentage of resection was calculated as follows: $[1$ - (postoperative tumor volume/preoperative tumor volume) $\times 100$, with gross-total resection (GTR) reflecting $>99 \%$ removal, and all other amounts classified as partial resection..$^{27}$ 


\section{Residual Bootstrap Q-Ball Tractography}

The HARDI data sets were corrected for movement and eddy-current distortions using the FMRIB Software Library (University of Oxford). The original gradient table was consequently rotated..$^{19}$ Diffusion imaging in Python (Dipy) software was used to estimate fractional anisotropy (FA) and for residual bootstrap q-ball fiber tracking. ${ }^{4,7}$

The signal was fit to spherical harmonics of even orders up to 4 , and orientation distribution functions (ODFs) were estimated from the data using the constant solid angle factor. ${ }^{30}$ Residual bootstrap tractography ${ }^{4}$ was applied by computing bootstrap ODFs at each voxel and identifying peaks in these ODFs. To identify the primary fiber orientation, ODF peaks were further refined by excluding peaks that were less than $45^{\circ}$ from a larger peak and small peaks with values less than one-fourth of the maximum of the ODF. The principal fiber orientations from the bootstrap ODFs provided the distribution of fiber tracking directions. Tracking was regulated using the following stopping criteria: FA threshold of 0.15 and maximum angle of $60^{\circ} .5$ Results were visualized using Trackvis (http://trackvis. org), and specific language pathways were reconstructed using a 2- or 3-ROI approach, depending on the pathway. The results were carefully inspected to exclude the possibility that susceptibility distortions may have affected the outcome of the tracking. ${ }^{17}$

In vivo diffusion MRI fiber tractography was performed by a trained neuroradiologist pre- and postsurgery to reconstruct white matter bundles belonging to dorsal phonological pathways (arcuate fasciculus [AF] or long segment of the perisylvian language network; superior longitudinal fasciculus component II [SLF-II], SLF component III [SLF-III], or anterior segment of the perisylvian language network; temporoparietal component of SLF [SLF-tp] or posterior segment of the perisylvian language network ${ }^{6}$ and ventral semantic streams (inferior frontooccipital fasciculus [IFOF], uncinate fasciculus [UF], inferior longitudinal fasciculus [ILF], and middle longitudinal fasciculus [MdLF]; Fig. 2). A representation of left-hemisphere ventral and dorsal stream pathways reconstructed for 10 control subjects and normalized to the Montreal Neurological Institute (MNI) space is furnished in Figs. 3 and 4.

We describe below the tracking approach for each white matter pathway studied.

\section{IFOF and UF}

Using an FA color map, a single-plane seed ROI was defined in the coronal plane passing through the anterior commissure, on which the anteroposterior tracts of the left external and extreme capsules were selected. The first target ROIs for the UF and IFOF were localized at the levels of the temporal and occipital lobes, respectively.,21 For both tracts, the ipsilateral frontal lobe was used as a second target ROI. Streamlines that passed through both target ROIs were retained.

\section{SLF-II and -III and AF}

A seed ROI was positioned in the coronal plane at the level of the isthmus of the corpus callosum, including a region of high anteroposterior anisotropy lateral to the central part of the lateral ventricle and the corona radiata. ${ }^{21}$

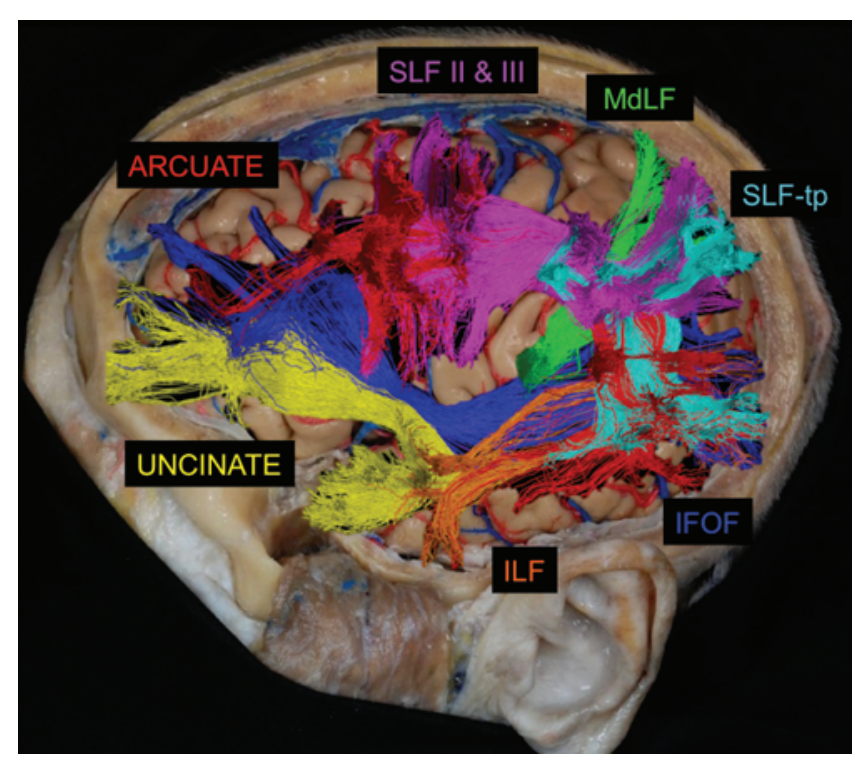

FIG. 2. Schematic representation of all evaluated tracts. IFOF = inferior frontooccipital fasciculus; ILF = inferior longitudinal fasciculus; Uncinate = uncinate fasciculus; MdLF = middle longitudinal fasciculus; SLF-tp = temporoparietal portion of SLF (or posterior segment of perisylvian language network ${ }^{6}$ ); Arcuate = arcuate fasciculus (or long segment of perisylvian language network ${ }^{6}$ ); SLF-II\&III = SLF components II and III (anterior segment of perisylvian language network ${ }^{6}$ ).

Target ROIs were selected as follows: in the angular gyrus for SLF-II, in the supramarginal gyrus for SLF-III, and on the axial peritrigonal plane at the level of the posterior middle and superior temporal gyri for the AF. The ipsilateral frontal lobe was used as a second target ROI. Streamlines that passed through both target ROIs were retained. Since the SLF-II and the SLF-III are difficult to separate in some subjects, these fasciculi were combined into the SLF-II\&III for the purposes of analysis.

\section{SLF-tp}

Using FA and an FA color map, the inferior parietal lobe (angular and supramarginal gyri) was seeded on an axial plane. The inferior parietal sulcus was used as an anatomical landmark to avoid including the superior portion of the parietal lobe. The target ROI was delineated to include the posterior middle and superior temporal gyri.

\section{MdLF}

A seed ROI was placed on the coronal plane passing through the posterior portion of the superior temporal lobe. ${ }^{20} \mathrm{~A}$ target coronal plane ROI perpendicular to the anterior commissure-posterior commissure (AC-PC) line was applied at the level where the parietooccipital and calcarine sulci meet, to select the posterior termination of the MdLF.

\section{ILF}

A single seed ROI was drawn in the temporal white matter on a coronal slice tangent to the anterior surface of the cerebral peduncles; only the lateral inferior white matter with an anteroposterior directionality was selected. A target ROI was placed at the level of the occipital lobe. ${ }^{21}$ 


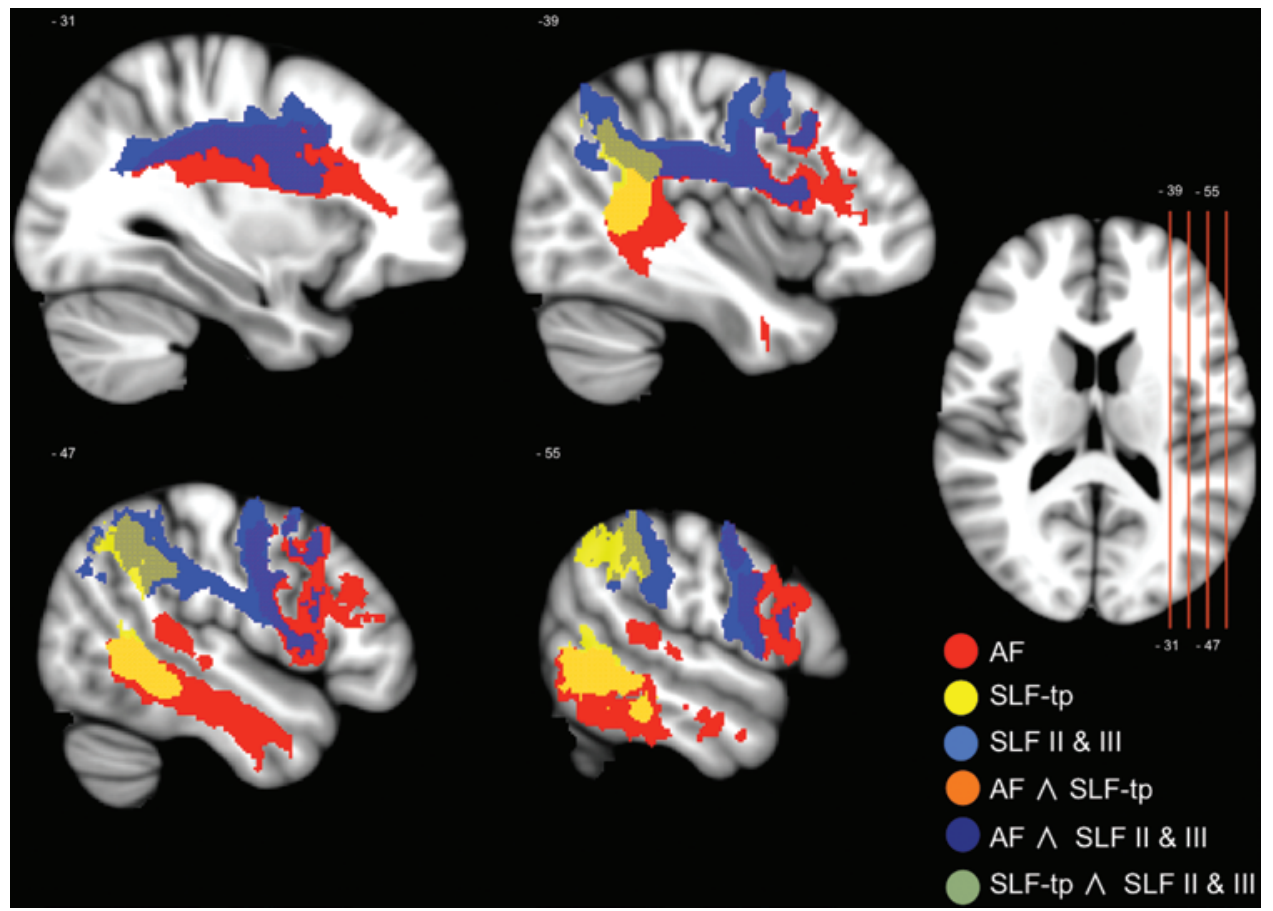

FIG. 3. Sagittal representation of left ventral stream pathways reconstructed for the 10 control subjects normalized to the MNI space (radiological orientation). Any MNI-space voxel that was included by the residual bootstrap tractography in at least $40 \%$ of the patients was considered part of a specific pathway. The MNI coordinates of the selected axial slices are reported on the right. Yellow indicates the SLF-tp (or posterior segment of arcuate ${ }^{6}$ ); red, the AF (or long segment of perisylvian language network ${ }^{6}$ ); blue, SLF-II\&III (anterior segment of perisylvian language network ${ }^{6}$ ); orange, green, and purple, overlapping of AF^SLF-tp, SLF$\|\&\| \|^{\wedge} S L F-t p$, and SLF-\|\& $\|^{\wedge} A F$, respectively.

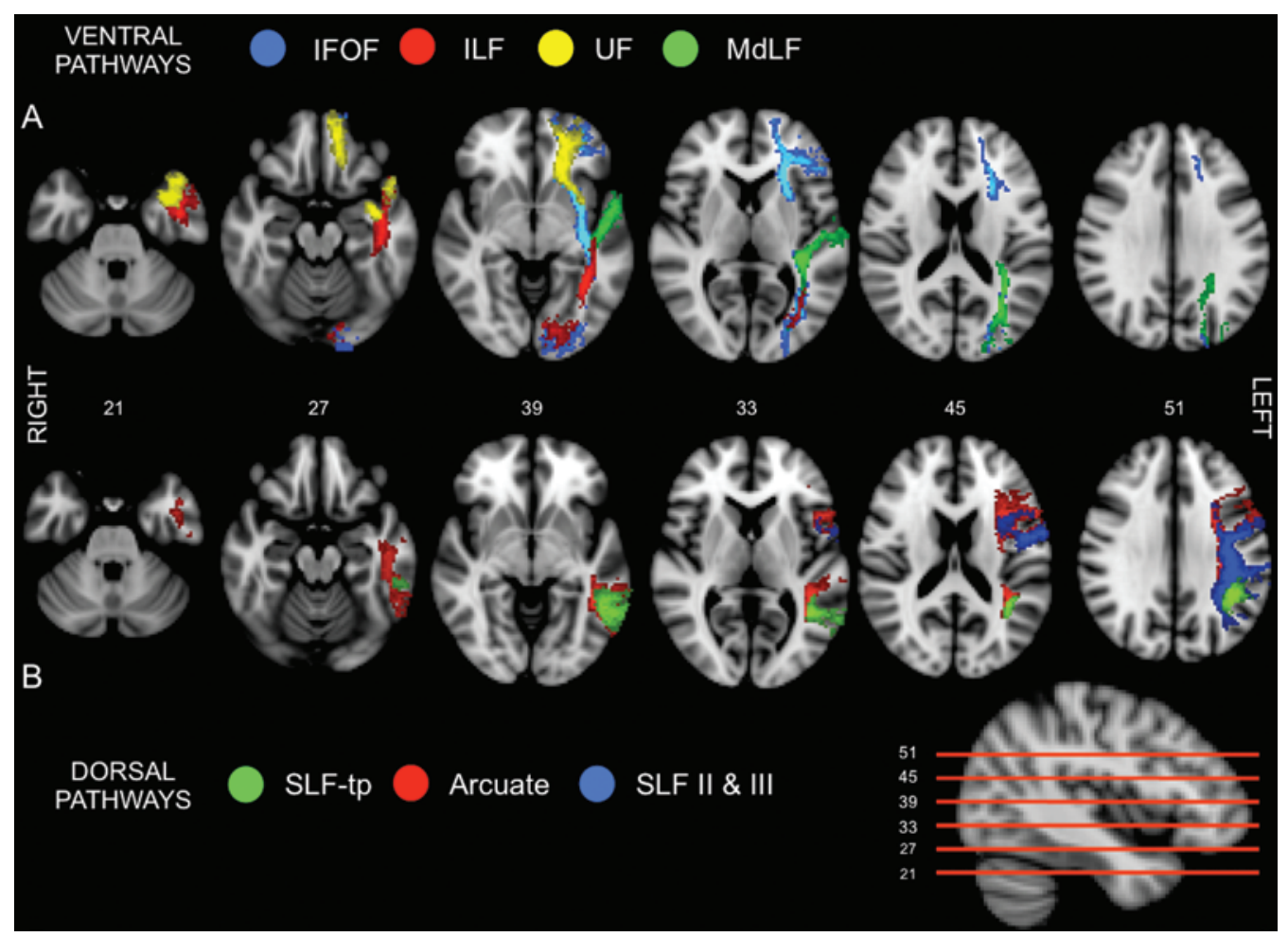

FIG. 4. Axial representations of the left ventral (A) and dorsal (B) pathways. Data were obtained in 10 healthy subjects normalized to the MNI space (radiological orientation). Any MNI-space voxel that was included by the residual bootstrap tractography in at least $40 \%$ of the patients was considered part of a specific pathway. The MNI coordinates of the selected axial slices are reported in the lower right corner. Ventral pathways: IFOF, ILF, UF, MdLF. Dorsal pathways: SLF-tp; AF; SLF-II\&III. 
For all previously described tracts, additional "NOT" ROIs (exclusion ROIs) were applied to remove obvious artifacts (common example: on the sagittal plane passing through the corpus callosum to exclude transhemispheric connections).

We performed tracking reconstruction on each subject's HARDI data, acquired both pre- and postsurgery. Seed ROIs for the postsurgery fiber tracking reconstruction were drawn using the location of the ROIs in the presurgery fiber tracking as reference. Reconstructed tracts in the subjects' native diffusion space were converted to density maps quantifying the number of streamlines passing through each voxel.

\section{Intraoperative Use of Tracking Results}

Presurgery FA maps of each patient were coregistered to the anatomical images using FMRIB Software Library linear and nonlinear transformations (FMRIB's FLIRT and FNIRT registration tools) so that each subject's presurgery fiber tracking results (as a binary mask) could be displayed on the anatomical images. The data were imported into the navigation system for intraoperative use.

\section{Rating Scores of Tracking Results}

Two operators independently evaluated the reconstructed tracts pre- and postsurgery, qualitatively rating the degree to which the reconstructed pathways were disturbed based on deviation from the expected tract location. The expected tract locations were based on healthy control tracts and homologous pathways contralateral to the tumor within the same subject. This rating of disturbance, based on altered fiber tractography density (AFTD), was determined by studying the reconstructed pathways relative to various images that included the FA color map and registered volumetric pre- and postsurgery T1-weighted and/or FLAIR sequences. The evaluation was made using 4 grades of tract involvement (no involvement, displaced, partially infiltrated, destroyed). We found no measurable difference between the no-involvement and displaced grades and, similarly, between the partially infiltrated and destroyed grades. Therefore, we combined the corresponding categories, which resulted in a binarized measure. The AFTD ratings (binary levels) were broadly classified as "not affected," with subcategories for unchanged (0) or displaced but otherwise normal appearing (1), or as "affected," with subcategories for partially interrupted (2) or completely interrupted (3). Interoperator concordance was assessed for the AFTD rating scale. For comparison with clinical deficits, differences were resolved by consensus. In the longitudinal evaluation, tract involvement was considered modified whenever a presurgery rating of $0-1$ increased to 2-3 after surgery.

\section{Clinical Language Deficit Correlations With Altered Fiber Tractography Density}

In cross-sectional analyses, we investigated the relationship between the presence of clinical language deficits $(C D=0$ or 1 , a binary variable for the absence or presence of deficits) and tract disruption status (binary AFTD $=0$ or 1 , a binary variable for not affected or affected). To model this relationship, we assumed the existence of a set of pathways in which damage to all members is necessary to cause clinical deficit. This tract set, termed the "minimally sufficient tract set" (MST set), is a subset of all tracts that are relevant to clinical language function, known as the "clinically relevant tract set" (CRT set). Possible redundancy and plasticity in the support of language function necessitates consideration of the scenario in which injury to multiple pathways may be required to elicit clinical language deficits. In the event of plasticity, the MST set will probably include multiple pathways; in acute cases, such as fast-growing tumors, damage to a single pathway may be sufficient to cause clinical deficits.

This model (CRT $\rightarrow$ minimal sufficient tracts $\rightarrow$ clinical function) limits the inferences from univariate correlations between tract status and clinical deficits. For example, a CRT could be damaged without the patient presenting with a deficit in the case of plasticity or redundancy. Direct univariate correlations may provide specificity but not sensitivity to functional pathways. Given the size and distribution limitations of our data set, we therefore chose to identify those pathways that associate directly with clinical deficits without laying claim to the identification of all pathways that may support language function.

\section{Patients Free of Clinical Language Deficit: Cross-Sectional Analyses}

There were no clinical deficits $(C D=0)$ in any of the low-grade glioma (LGG) patients, and only $15 \%$ of the high-grade glioma (HGG) patients presented with clinical deficit $(C D=1)$. From such data, we cannot infer the true association between tract status and clinical deficit, but we can identify those pathways that are more likely to be preserved in the absence of clinical deficits. We can infer that tracts that are highly preserved in this subcohort may belong to the CRT set, while those that are significantly affected are unlikely to (by themselves) constitute an MST. Therefore, these data are capable of providing candidate tracts for the CRT set based on their action as a member of the MST set, but cannot be used to establish a tract as clinically irrelevant.

For each pathway and tumor grade (LGG and HGG), the fraction of patients with affected tracts (binary AFTD $=1$ ) was computed. The probability that this fraction was greater than or equal to 0.5 was determined based on a binomial model. Pathways with $\mathrm{p}<0.00625$ (Bonferroni correction for multiple comparisons across 8 pathways) were considered tracts that are preserved in the absence of clinical deficits.

\section{Predicting Long-Term Clinical Deficits}

Since there were no LGG patients with clinical language deficits at the long-term time point, the ability of tract status at discharge to predict long-term clinical deficits was evaluated in HGG patients only. The prediction of long-term clinical deficits ( $C D=0$ or 1$)$ using the tractaffected status at discharge (AFTD $=0$ or 1 ) was computed for the pathways found to be significantly preserved in those patients with normal clinical function presurgically. We applied a nominal logistic regression model first to all HGG patients with clinical deficits at postsurgical Days 
3-5 (21 patients) and then only to those with clinical deficits at discharge (11 patients).

\section{Results}

\section{Reliability of Fiber Tracking Rating in Patients}

Interoperator agreements on the sublevels of AFTD tract ratings (4 levels) were $96 \% \pm 3 \%$ (mean \pm standard deviation) for presurgery data and $98 \% \pm 2 \%$ for postsurgery data. For the binary AFTD (affected vs not affected), the interoperator agreements were $98 \% \pm 2 \%$ for presurgery data and $99 \% \pm 1 \%$ for postsurgery data. Differences were resolved by consensus.

\section{Clinical Profile}

Of the 78 cases treated with awake left-hemisphere resection with ISM techniques for a WHO Grade II or higher tumor, 35 met our study inclusion criteria. Reasons for excluding the remaining patients were as follows: right-hemisphere surgery ( 27 of 43), previous resection (7 of 43), or postoperative HARDI acquisitions not available (9 of 43). In the 35 patients ( 14 females, 21 males, mean age $46 \pm 15$ years, median age 49 years) eligible for this study, tumors included WHO Grade II (14 cases [40\%]: 7 oligoastrocytoma, 5 diffuse astrocytoma, 1 oligodendroglioma, 1 ependymoma), WHO Grade III (11 cases [31\%]: 6 anaplastic oligodendroglioma, 4 anaplastic astrocytoma, 1 anaplastic pleomorphic xanthoastrocytoma), and WHO Grade IV (10 cases [28\%] of glioblastoma multiforme). Ninety-four percent (33/35) of the patients were right-handed. Magnetoencephalographic imaging for the language laterality index indicated left-hemisphere dominance in $74.3 \%(26 / 35)$ of the patients, right-hemisphere dominance in 1 patient, and bilaterality in $11.4 \%$ (4/35) of patients (Table 1$)$. In 4 patients, laterality index results were not conclusive.

\section{Language Morbidity Profile}

Of the 35 patients, 28 (80\%) had normal preoperative language function, which was preserved at the long-term follow-up. Of these 28 patients, 5 (18\%) had short-term language dysfunction at the time of discharge but fully recovered within 3 months. Of the 35 patients, 3 (9\%) had presurgery language dysfunction that persisted permanently after surgery (final clinical assessment performed at 6 months), 4 (11\%) developed new postsurgery language dysfunction that persisted after 6 months, and $5(14 \%)$ developed transient language dysfunction that had completely resolved by the 3- to 6-month follow-up. The majority of patients (23 [66\%]) maintained normal pre- and postoperative language function. Complete language morbidity profiles are shown in Table 2.

\section{Tumor Volumetric Analysis}

The average percentage of lesion resection was $88 \%$ $\pm 15 \%$ in patients with LGGs and $92 \% \pm 11 \%$ in patients with HGGs. In the LGG subgroup, 4 (28.6\%) of 14 individuals were judged to have GTR (resection $>99 \%$ ). Of those with partial resections, $21.4 \%$ had $90 \%-99 \%$ of the tumor removed, $35.7 \%$ had $80 \%-90 \%$ removed, and $14.3 \%$ had $45 \%-80 \%$ removed. In the HGG subgroup, 11 (52.4\%) of 21 individuals were judged to have GTR. Of those with partial resections, $14.3 \%$ had $90 \%-99 \%$ removed, $9.5 \%$ had $80 \%-90 \%$ removed, and $23.8 \%$ had $66 \%-80 \%$ removed.

\section{Tracking Results}

Eight white matter pathways were reconstructed preoperatively with different levels of tumor involvement determined by the AFTD rating in each of the 35 patients (total of 280 tracts). Between the pre- and postsurgery tract evaluations, $81 \%$ (226 of 280) of the tracts had no change in AFTD score, $16 \%(44 / 280)$ had a worse AFTD score, and $4 \%(10 / 280)$ had an improved AFTD score. Significant worsening (null/mild to moderate/severe AFTD changes) occurred in $9 \%$ of the tracts $(26 / 280)$.

\section{Preservation of AF and SLF-tp in Patients With No Presurgery Deficits}

Evaluation at a cross-sectional level of presurgery data showed that 32 of 35 patients had no clinical deficits, with only 3 of 18 HGG subjects showing language deficits. Analysis of the pathway involvement (Table 3 ) showed that some tracts are affected in the absence of language deficits, suggesting that clinical language assessments are not sensitive to the function served by these pathways or that redundancy for language support was present. Conversely, the 4 tracts that were preserved (AF, SLF-tp, ILF, and MdLF) are candidates for tracts that may relate to clinical language deficits. Note that only AF and SLF-tp survived Bonferroni correction for significance $(p<0.00625)$.

\section{Longitudinal Analysis}

Twelve of the 35 patients (11 HGG, 1 LGG) had deficits at discharge. Five (Cases 6, 19, 21, 23, 26) of the 32 patients with no language deficit at the presurgery time point showed transient language deficits at discharge (within 3 days), which were resolved at the long-term follow-up (Fig. 5). All were HGG cases except for Case 21 (LGG).

Seven HGG patients with clinical deficits at discharge continued to have deficits at the long-term follow-up (> 3 months). Three of these patients had language deficits presurgery, which did not change between the pre- and postsurgery time points (Fig. 6). Four of the HGG patients experienced the onset of a new long-term language deficit; only 1 of these patients (Case 22) had significant (AFTD change from 0 to 1 ) worsening of tracts (AF and SLF-tp; Fig. 7). Tract changes in these 4 subjects are reported in Table 4.

\section{Predicting Long-Term Language Deficits in Patients With Deficits at Discharge}

We evaluated the ability of postsurgery predischarge AFTD tract status to predict long-term clinical deficits. Given the preservation of the SLF-tp and AF in patients without deficits presurgically, as described above, we tested the predictive value of these pathways to determine which of the 11 HGG patients with deficits at discharge would continue to have long-term deficits and which would have resolution of their deficits. The nominal logistic regression determined a significant model $(\mathrm{p}=0.005)$. The positive predictive and sensitivity values were both $86 \%$, and the 
TABLE 1. Demographics and clinical information for 35 patients in the study cohort

\begin{tabular}{|c|c|c|c|c|c|}
\hline Case No. & Histology & WHO Grade & Age (yrs) & Language Laterality Index & Handedness \\
\hline 1 & Anaplastic oligodendroglioma & III & 40 & $\mathrm{Lt}$ & $\mathrm{Rt}$ \\
\hline 2 & Oligodendroglioma & II & 44 & $\mathrm{Lt}$ & Rt \\
\hline 3 & Glioblastoma & IV & 55 & $\mathrm{Lt}$ & Rt \\
\hline 4 & Infiltrative astrocytoma & II & 30 & $\mathrm{Lt}$ & Rt \\
\hline 5 & Anaplastic astrocytoma & III & 54 & $\mathrm{Lt}$ & Rt \\
\hline 6 & Glioblastoma & IV & 62 & $\mathrm{Lt}$ & Rt \\
\hline 7 & Ependymoma & II & 21 & Bilat & Rt \\
\hline 8 & Anaplastic oligoastrocytoma & III & 55 & Lt & Rt \\
\hline 10 & Glioblastoma & IV & 59 & $\mathrm{Lt}$ & Rt \\
\hline 11 & Anaplastic oligodendroglioma & III & 49 & Lt & $\mathrm{Rt}$ \\
\hline 12 & Oligoastrocytoma & II & 51 & Lt & $\mathrm{Rt}$ \\
\hline 14 & Oligoastrocytoma & II & 48 & Bilat & $\mathrm{Rt}$ \\
\hline 15 & Diffuse astrocytoma & II & 18 & Lt & Rt \\
\hline 16 & Oligoastrocytoma & II & 33 & Lt & Rt \\
\hline 17 & Glioblastoma & IV & 75 & - & Rt \\
\hline 19 & Anaplastic oligodendroglioma & III & 63 & Lt & Rt \\
\hline 20 & Oligoastrocytoma & II & 56 & $\mathrm{Rt}$ & Rt \\
\hline 21 & Infiltrating astrocytoma & II & 54 & Lt & Rt \\
\hline 22 & Glioblastoma & IV & 62 & Lt & $\mathrm{Lt}$ \\
\hline 23 & Glioblastoma & IV & 61 & Bilat & $\mathrm{Rt}$ \\
\hline 24 & Oligoastrocytoma & II & 20 & Bilat & Rt \\
\hline 25 & Infiltrating astrocytoma & $\|$ & 21 & Lt & Rt \\
\hline 26 & Anaplastic astrocytoma & III & 38 & $\mathrm{Lt}$ & Rt \\
\hline 27 & Glioblastoma & IV & 59 & $\mathrm{Lt}$ & Rt \\
\hline 28 & $\begin{array}{l}\text { Anaplastic pleomorphic xantho- } \\
\text { astrocytoma }\end{array}$ & III & 43 & $\mathrm{Lt}$ & Rt \\
\hline 29 & Glioblastoma & IV & 68 & - & Rt \\
\hline 30 & Diffuse astrocytoma & II & 39 & Lt & $\mathrm{Rt}$ \\
\hline 31 & Anaplastic oligodendroglioma & III & 36 & $\mathrm{Lt}$ & Rt \\
\hline 32 & Oligoastrocytoma & II & 29 & Lt & $\mathrm{Rt}$ \\
\hline 33 & Anaplastic astrocytoma & III & 22 & $\mathrm{Lt}$ & $\mathrm{Lt}$ \\
\hline 34 & Glioblastoma & IV & 51 & - & $\mathrm{Rt}$ \\
\hline 35 & Anaplastic oligodendroglioma & III & 59 & $\mathrm{Lt}$ & $\mathrm{Rt}$ \\
\hline 36 & Oligoastrocytoma & II & 33 & $\mathrm{Lt}$ & Rt \\
\hline 37 & Glioblastoma & IV & 64 & - & $\mathrm{Rt}$ \\
\hline 38 & Anaplastic astrocytoma & III & 49 & $\mathrm{Lt}$ & $\mathrm{Rt}$ \\
\hline
\end{tabular}

negative predictive values and specificity values were both $75 \%$. If the SLF-tp and AF were affected on postsurgery analysis, patients tended to have long-term deficits lasting more than 3 months. However, if these tracts were not affected during surgery, only 1 of 4 patients with short-term language disability presented a long-term ( $>3$ months) deficit (Table 5).

\section{Discussion}

We report on the development and implementation of pre- and postoperative mapping of putative language pathways with high angular resolution diffusion MRI for neurosurgical planning, intraoperative mapping, and postsurgical prediction of deficit resolution. These meth- ods, including the rating of fiber tract injury, were found to be highly reliable. Our results suggest that dorsal pathways (AF and SLF-tp) are preserved in patients without language deficits, and their integrity may be predictive of whether a postoperative language deficit will resolve.

\section{Defining Language Pathways With Diffusion MRI: Accuracy, Reliability, and Rating}

Currently, DTI tractography is the most commonly used method of mapping white matter in the daily clinical and research setting worldwide. Even though fiber tractography with the DTI model has contributed greatly to our knowledge of fiber pathways, it suffers from several serious shortcomings in resolving the extent, origin, and 
TABLE 2. Language morbidity profile

\begin{tabular}{|c|c|c|c|}
\hline No. of Patients & $\begin{array}{l}\text { Presurgery Language } \\
\text { Assessment }\end{array}$ & $\begin{array}{l}\text { Discharge Language } \\
\text { Assessment }\end{array}$ & Long-Term FU (>3 mos postsurgery) \\
\hline $23(66 \%)$ & No deficit & No deficit & No deficit \\
\hline $5(14 \%)$ & No deficit & $\begin{array}{l}\text { New-onset language } \\
\text { deficit }\end{array}$ & Resolved language deficit \\
\hline $4(11 \%)^{*} \dagger$ & No deficit & $\begin{array}{l}\text { New-onset language } \\
\text { deficit }\end{array}$ & $\begin{array}{l}\text { Persistent language deficit; severe postop aphasia; word substitutions in repetition; } \\
\text { absence of naming, counting, or reading deficits; severe expressive aphasia; } \\
\text { word-finding difficulties; normal speech fluency, object naming, repeat, count, \& } \\
\text { calculation }\end{array}$ \\
\hline $3(8.6 \%) \dagger$ & Language deficit & $\begin{array}{l}\text { Persistent language } \\
\text { deficit }\end{array}$ & $\begin{array}{l}\text { Persistent language deficit; stable expressive \& receptive aphasia; stable expressive } \\
\text { aphasia; stable expressive aphasia }\end{array}$ \\
\hline
\end{tabular}

cortical termination of white matter pathways. Novel diffusion model tractography algorithms based on HARDI have been developed in recent years. In particular, residual bootstrap q-ball has been shown to improve the resolution of white matter fiber tractography, especially in crossing regions. ${ }^{5,7}$ Nevertheless, the application of these advanced tractography methods has generally been confined to research. At our institution, we have successfully implemented residual bootstrap probabilistic q-ball tractography in presurgery planning for language pathways in patients with brain tumor. We were able to apply this novel technique consistently in a large cohort of tumor patients (more than 300 since January 2012). The HARDI data were acquired in almost all subjects the day before surgery. Especially in HGG patients, the timing of MRI is crucial to minimize possible changes between MRI data acquisition and the actual surgery.

Multiple pathways belonging to either the dorsal or ventral streams ${ }^{16}$ were reconstructed and uploaded to the neuronavigation system to be combined with intraoperative cortical and subcortical stimulation. White matter pathway reconstruction was also performed postsurgery, based on HARDI acquisitions within 1-2 days of surgery. The HARDI data acquired postsurgery were moderately high quality and there was no significant postoperative swelling, so white matter tractography could be performed on all of the tumor patients. We could then compare the pre- and postsurgery results to identify pathway changes related to the surgical intervention.

Independent of the algorithm applied, tractography data must be carefully reviewed and evaluated for an accurate intraoperative application. This represents a challenge for both the surgeon and the tractography operators. The importance of anatomical knowledge and experience is crucial, as is establishing the reliability of the tract.

It is important to mention that we are discussing in vivo virtual reconstructions, which do not necessarily represent real anatomy and therefore need anatomical validation. We have recently shown that q-ball tractography can represent the more complex connectivity of these pathways than can previous technologies, validated by anatomical dissection. ${ }^{7}$ Even those pathways (such as the AF/SLF) that are well established and anatomically proven seem to present a more complex structure when tracked using HARDI compared with DTI. We reconstructed white matter pathways belonging to either the dorsal or ventral language streams. ${ }^{16}$ For the first time in brain tumor patients, we independently evaluated the multiple components of the AF/SLF complex, previously treated as a single unit, ${ }^{1,2}$ and extended the evaluation to other pathways such as the MdLF and ILF.

Though the anatomical structure of the pathways reported herein is in need of validation through dissection, this reconstruction procedure was tested in more than 300 patients. The q-ball-based structural connectivity of the pathways we studied and described in this paper was relatively consistent across this large group of subjects (not shown).

In this paper, we also applied and proposed a rating scale of the tracking results that differentiated the level to which a pathway has been infiltrated or interrupted, a scale similar to those employed previously. ${ }^{2}$ The main challenge inherent to this rating scale was the ability to distinguish tracts primarily dislocated from tracts partially infiltrated

TABLE 3. Identification of tracts that were highly preserved in the absence of clinical deficits*

\begin{tabular}{ccc}
\hline & \multicolumn{2}{c}{ Presurgery Tract Ratings, \% Not Affected } \\
\cline { 2 - 3 } Pathways & All Patients $(\mathrm{n}=32)$ & Patients w/ HGG $(\mathrm{n}=18)$ \\
\hline SLF-II & $53 \%$ & $44 \%$ \\
\hline SLF-III & $44 \%$ & $33 \%$ \\
\hline AF & $78 \% \dagger$ & $89 \% \dagger$ \\
\hline SLF-tp & $91 \% \dagger$ & $83 \% \dagger$ \\
\hline ILF & $81 \% \dagger$ & $78 \%$ \\
\hline MdLF & $88 \% \dagger$ & $78 \%$ \\
\hline IFOF & $69 \%$ & $56 \%$ \\
\hline UF & $69 \%$ & $67 \%$ \\
\hline
\end{tabular}

* Percentage of patients with preserved tracts $(A F T D=0)$ for each pathway within the subgroup with no language deficit presurgically. Statistical testing was performed with the null hypothesis that the percent affected was greater than or equal to $50 \%$.

$\dagger$ The null hypothesis was rejected at a Bonferroni corrected $p$ value $<0.006$. 

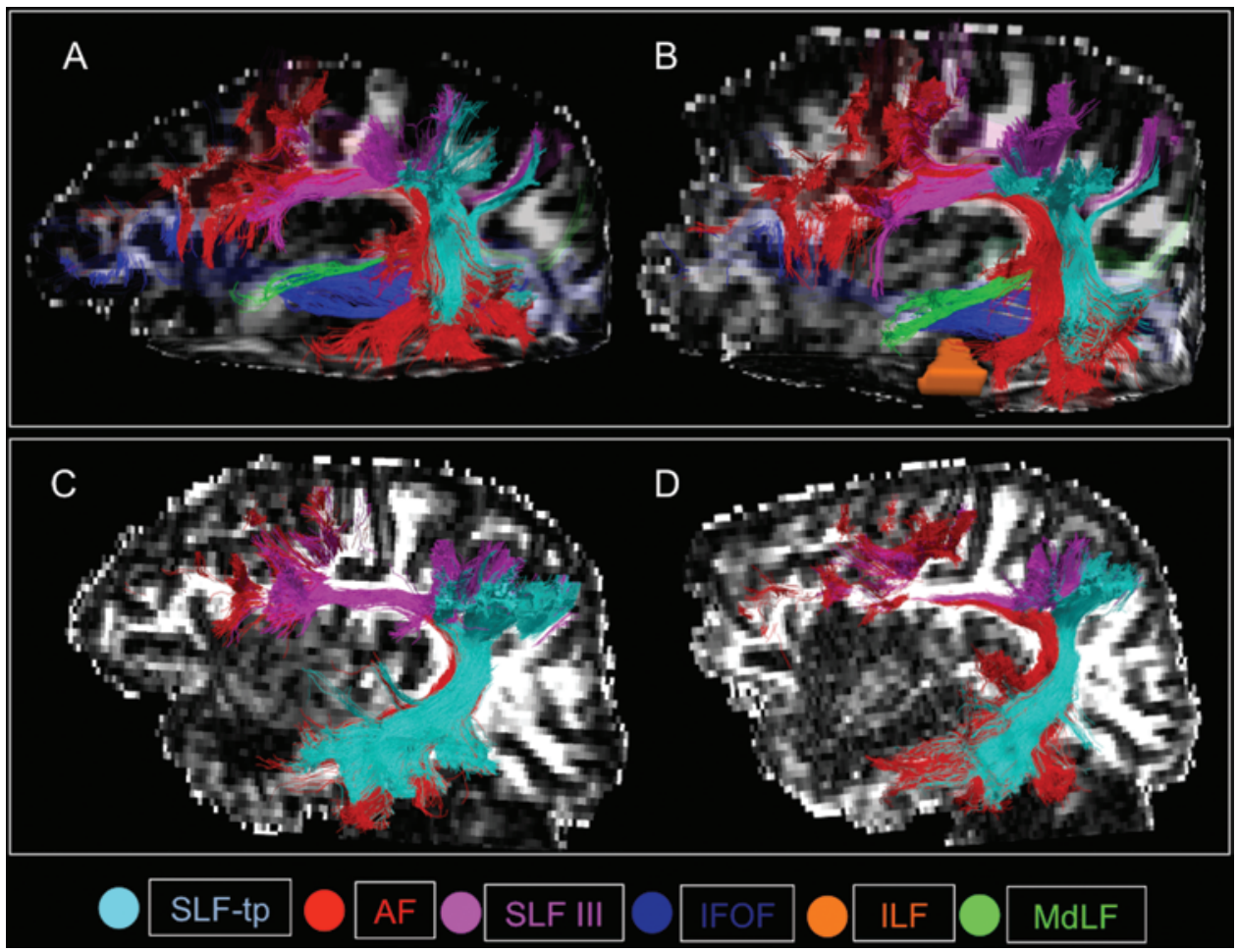

FIG. 5. Tracking results in 2 HGG patients with transient postsurgical language deficits. Four HGG patients experienced the onset of transient language deficit at discharge (within 3 days), which had resolved at the long-term follow-up. This figure shows the results in 2 of these cases. Case 23. Tracking results presurgery (A) and postsurgery (B). The patient showed significant involvement (AFTD > 1) of the UF and ILF. The pre- and postsurgery tracking results generally overlapped, except for the IFOF, whose inferior portion was partially resected. The surgical approach is depicted in orange. Case 26. Tracking results presurgery (C) and postsurgery (D). The patient showed significant involvement (AFTD >1) of all ventral stream pathways with no significant AFTD change following surgery.

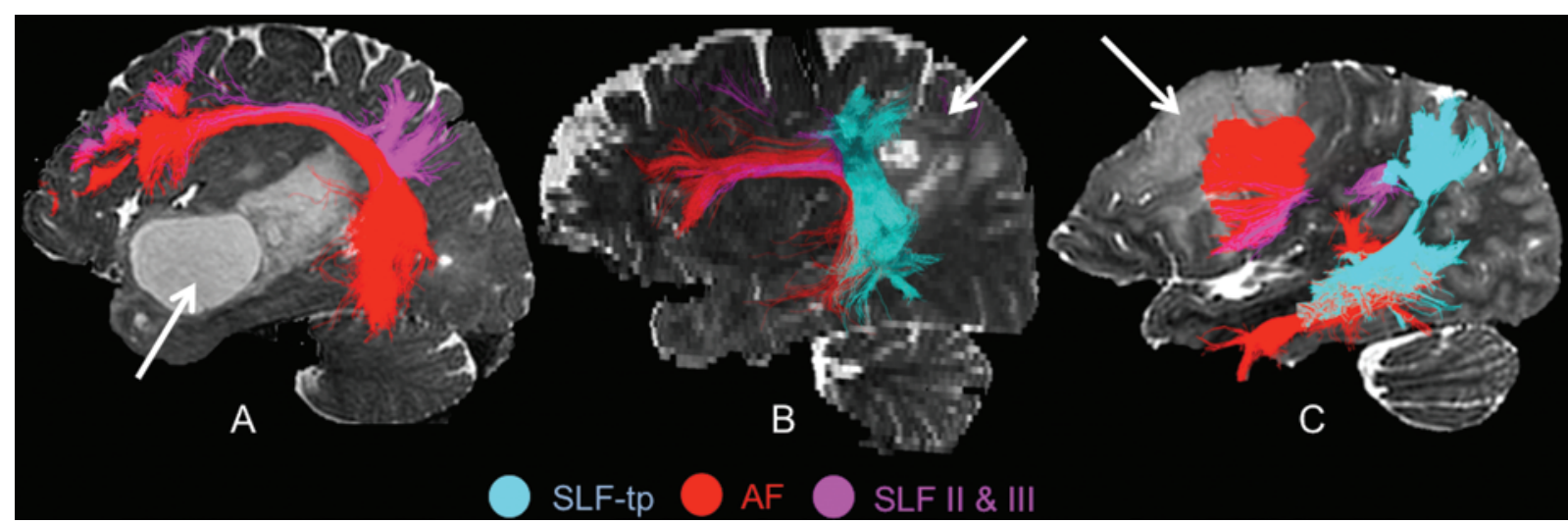

FIG. 6. Cases with presurgical language deficit. We show the dorsal pathway status in 3 HGG cases with a presurgical language deficit that did not change between pre- and postsurgery time points. White arrows indicate tumor location. Case 29. The patient had a glioblastoma involving the frontotemporoinsular region (A) as well as a language deficit presurgery. The SLF-III (or anterior portion of perisylvian language network ${ }^{6}$ ) and SLF-tp (or posterior segment of perisylvian language network ${ }^{6}$ ) were severely affected. The AF (or long segment of perisylvian language network ${ }^{6}$ ) and SLF-II appeared to be preserved and, at most, dislocated by the tumor $(A F T D=1)$. Note that the SLF pictured is predominantly the SLF-II. All of the ventral stream pathways were severely affected by tumor and could not be reconstructed. Case 34. The patient had a glioblastoma involving the inferior parietal lobule (B) as well as a presurgical language deficit with involvement of the AF and SLF-tp posteriorly. Regarding the ventral stream pathways, all tracts were successfully reconstructed (not shown) except the MdLF (not shown), which was severely affected by tumor. Case 31. The patient had an anaplastic oligodendroglioma involving the frontoinsular region (C) and a presurgical language deficit. The SLF-III and AF were severely affected. Regarding the ventral stream pathways, all tracts were successfully reconstructed except the IFOF (not shown), which was severely affected by tumor. 


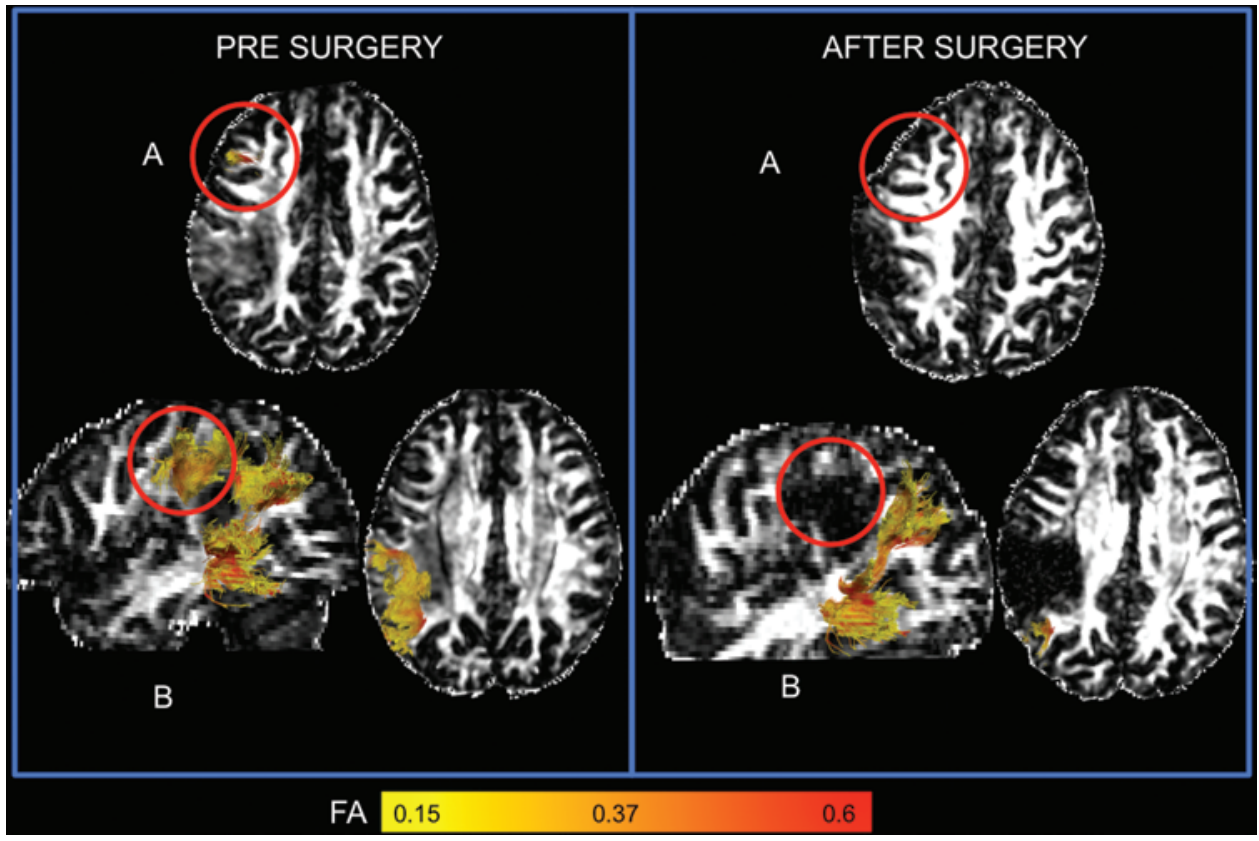

FIG. 7. Case 22. The patient experienced the onset of a new long-term language deficit. This was the only patient (out of 4) whose onset of language dysfunction after surgery could be correlated with changes in tracking results between pre- and postsurgery. The caudal middle frontal termination of the AF $(A)$ and the supramarginal portion of the SLF-tp (B) could not be tracked postsurgery.

or interrupted. Although based purely on a qualitative evaluation, these rating scores for fiber pathway damage were highly reproducible across operators and provided significant correlations with language deficits.

White matter pathway reconstruction is crucial to understanding the structure and changes occurring both within the borders of and in the zone surrounding brain tumors. ${ }^{10}$ We also have to consider the high plasticity potential of the human brain, particularly that of gray matter. ${ }^{13}$ White matter (especially projection and association pathways) has a critical role in brain function. Contrary to gray matter structures, these white matter pathways show a very low intersubject variability and, more importantly, very limited reorganization and plasticity. ${ }^{13}$ These white matter structures often come to represent the limits of the tumor resection, as many surgeons are hesitant to risk introducing irreversible clinical dysfunction postsurgery. ${ }^{13}$ Integrity of association and projection pathways also plays a crucial role in the development of gray matter plasticity, representing the "infrastructure" on which brain reorganization occurs. ${ }^{13}$

Recently, the existence of a direct connection between Wernicke's and Broca's areas in the human brain has been questioned..$^{13,22}$ Using anatomical cortex-sparing fiber dissection, Martino and colleagues showed that the AF seems to be posteriorly connected to the inferior and middle temporal gyri. ${ }^{22}$ In particular, they did not observe connections to the superior temporal gyrus but instead described connections to the precentral gyrus through the anterior segment of the SLF. In the current study, we consider the $\mathrm{AF}$ to be the collection of pathways connecting the frontal lobe to the temporal lobe, including the anterior segment of the SLF as described by Martino et al. In our q-ball tracking experience in over 300 patients with primary gliomas, this bundle seems to belong to the AF and to be distinct from the SLF subcomponent connected to the

TABLE 4. Patients with postsurgery language deficit at discharge*

\begin{tabular}{cllll}
\hline Case No. & WHO Grade, Lesion Type & New Long-Term Language Deficit & Newly Affected Pathways & AFTD Change \\
\hline 3 & IV, glioblastoma & Severe aphasia & SLF-II & High to high: 2 to 3 \\
\hline 5 & III, anaplastic astrocytoma & Word substitutions in repetition & SLF-tp† & High to high: 2 to 3 \\
\cline { 3 - 4 } & IV, glioblastoma & Severe expressive aphasia & ILF† & Low to low: 0 to 1 \\
\hline 10 & IV, glioblastoma & Word-finding difficulties & None & None \\
\hline
\end{tabular}

* Tracking rating scale (AFTD) findings for the 4 patients with new-onset language deficit postsurgery and persisting at the 3-month follow-up. The tracts that show the worse rating postsurgery as compared to presurgery (AF, SLF-tp) would therefore be candidates for functional pathways related to the clinical language assessments. The rating scale changes between pre- and postsurgery are reported in the far right column.

$\dagger$ Data not imported into the Brainlab navigation system for intraoperative use. 
TABLE 5. Impact of SLF-tp and AF involvement on long-term language disability

\begin{tabular}{lcc}
\hline & \multicolumn{2}{c}{ No. of Patients } \\
\cline { 2 - 3 } Postsurgical Status & $\begin{array}{c}\text { Language Deficit at } \\
\text { Long-Term FU } \\
(>3 \text { mos })\end{array}$ & $\begin{array}{c}\text { No Language Deficit } \\
\text { at Long-Term FU } \\
(>3 \text { mos })\end{array}$ \\
\hline SLF-tp \&/or AF affected & 6 & 1 \\
\hline SLF-tp \& AF not affected & 1 & 3 \\
\hline
\end{tabular}

supramarginal gyrus (SLF-III). This is also evident from the FA maps, on which the white matter of the superior temporal gyrus and the supramarginal gyrus are clearly demarcated. From our experience tracking the SLF-tp (or posterior segment of SLF), ${ }^{22}$ we have noticed that this pathway connects the supramarginal and angular gyri to the posterior portions of the middle and inferior temporal lobe areas and, less frequently, to the superior temporal gyrus (Figs. 2 and 3 ).

The relative lack of postsurgery language deficits in our cohort, especially considering the location of the tumor in presumed language areas, is a promising result supporting the intraoperative use of these language tracts. Our cohort was histopathologically heterogeneous, including both LGG and HGG. The LGG subgroup did not show language deficits either presurgery or at the long-term followup. Examining the subgroup of patients with no language deficit pre- (Table 3 ) or postsurgery, we found 4 tracts (AF, SLF-tp, MdLF, and ILF) that were relatively preserved. Of these tracts, only the AF and SLF-tp were preserved significantly above chance and thus were designated as candidates for critical language network components influencing language deficits.

Despite the limitations inherent to a small sample size, our preliminary data highlighted for the first time a possible prognostic value of the postsurgery tracking assessment for long-term language function. For patients with a language deficit after surgery, the preservation of dorsal pathways (SLF-tp and AF, in particular) may represent a positive prognostic factor of recovery over the long term (Figs. 5 and 7).

Altogether, these preliminary findings provide evidence for the importance of the AF and SLF-tp to clinical language deficits, though they do not rule out the possible importance of ILF and MdLF. These data also suggest that isolated damage to the other pathways (SLF-II, SLF-III, IFOF, and UF) does not result in clinical deficits, as these pathways are affected in the absence of language deficits. It should be noted that clinical language assessments may not be sensitive to the functions served by these pathways.

Our results indicate that the dorsal subcortical language tracts should be mapped during the course of surgery to possibly identify these tracts and preserve them, as is routinely done for essential cortical sites. Cortical and subcortical eloquent structures are equally important because they may carry different language components of speech. Both should be independently mapped and preserved if identified.

We believe that DTI is no longer the de facto standard for fiber tracking. It may be the standard for computing metrics for in-group studies, but the inability of DTI to model crossing fibers cannot be ignored in fiber tracking. We believe that our results better represent the brain connectivity of white matter pathways and, if used correctly, can be an important tool for intraoperative use and assessment of patients pre- and postsurgery to correlate and understand the spectrum of clinical deficits in brain tumor patients.

\section{Limitations}

There are several clinical and technical limitations to this study. First is the relatively small number of patients in whom the tracking versus language correlation was tested. As previously discussed, it is possible that the clinical evaluation was not sensitive to language function modifications in LGG cases. The lack of clinical findings in the LGG group, as well as the limited correlation with the ventral stream pathways, may be due to plasticity or deformation by a slow-growing expansive lesion. Alternatively, more complex testing may have better characterized certain language deficits, for example, those related to higherlevel language comprehension.

The possibility of evaluating the predictive value of our assessment (AFTD) of white matter fiber involvement in brain tumors for long-term language deficits was limited by the small number of cases with clinical deficits, none of which were in the LGG group. Moreover, a multimodal approach (including functional techniques) may explain the occurrence of language deficits more precisely than would a "structural" connectivity approach. The bootstrap q-ball tracking in presurgery planning is an ongoing project, which is still in the process of methodological development. Because of the limitations underlying diffusion imaging and tractography algorithms, we consider confirmation via postmortem anatomical dissection to be an important step toward the validation of the anatomy of white matter language pathways.

\section{Conclusions}

We demonstrate the successful application of q-ball tracking in presurgery planning for language pathways in brain tumor patients and in assessing white matter tract integrity postoperatively. Our results suggest the importance of dorsal stream tract preservation (AF and SLF-tp) to reduce language deficits in patients undergoing glioma resection and the potential prognostic value of using tract integrity on postoperative imaging to determine if a patient with a postresection deficit has the potential to recover language function.

\section{Acknowledgments}

This work was supported by National Institutes of Health Grant Nos. R01 NS066654 (R.G.H.) and P50 CA097257 (M.S.B.) and by the Department of Defense through the National Defense Science \& Engineering Graduate Fellowship program (K.M.J.).

\section{References}

1. Bello L, Castellano A, Fava E, Casaceli G, Riva M, Scotti $\mathrm{G}$, et al: Intraoperative use of diffusion tensor imaging fiber tractography and subcortical mapping for resection of glio- 
mas: technical considerations. Neurosurg Focus 28(2):E6, 2010

2. Bello L, Gambini A, Castellano A, Carrabba G, Acerbi F, Fava E, et al: Motor and language DTI Fiber Tracking combined with intraoperative subcortical mapping for surgical removal of gliomas. Neuroimage 39:369-382, 2008

3. Berman JI, Berger MS, Mukherjee P, Henry RG: Diffusiontensor imaging-guided tracking of fibers of the pyramidal tract combined with intraoperative cortical stimulation mapping in patients with gliomas. J Neurosurg 101:66-72, 2004

4. Berman JI, Chung S, Mukherjee P, Hess CP, Han ET, Henry RG: Probabilistic streamline q-ball tractography using the residual bootstrap. Neuroimage 39:215-222, 2008

5. Bucci M, Mandelli ML, Berman JI, Amirbekian B, Nguyen C, Berger MS, et al: Quantifying diffusion MRI tractography of the corticospinal tract in brain tumors with deterministic and probabilistic methods. Neuroimage Clin 3:361-368, 2013

6. Catani M, Jones DK, ffytche DH: Perisylvian language networks of the human brain. Ann Neurol 57:8-16, 2005

7. Caverzasi E, Papinutto N, Amirbekian B, Berger MS, Henry RG: Q-ball of inferior fronto-occipital fasciculus and beyond PLoS One 9:e100274, 2014

8. Chaichana KL, Halthore AN, Parker SL, Olivi A, Weingart $\mathrm{JD}$, Brem H, et al: Factors involved in maintaining prolonged functional independence following supratentorial glioblastoma resection. Clinical article. J Neurosurg 114:604-612, 2011

9. Chang EF, Clark A, Smith JS, Polley MY, Chang SM, Barbaro NM, et al: Functional mapping-guided resection of low-grade gliomas in eloquent areas of the brain: improvement of long-term survival. Clinical article. J Neurosurg 114:566-573, 2011

10. Cruz Júnior LC, Sorensen AG: Diffusion tensor magnetic resonance imaging of brain tumors. Neurosurg Clin N Am 16:115-134, 2005

11. Dick AS, Tremblay P: Beyond the arcuate fasciculus: consensus and controversy in the connectional anatomy of language. Brain 135:3529-3550, 2012

12. Duffau H: Does post-lesional subcortical plasticity exist in the human brain? Neurosci Res 65:131-135, 2009

13. Duffau H, Herbet G, Moritz-Gasser S: Toward a pluri-component, multimodal, and dynamic organization of the ventral semantic stream in humans: lessons from stimulation mapping in awake patients. Front Syst Neurosci 7:44, 2013

14. Henry RG, Berman JI, Nagarajan SS, Mukherjee P, Berger MS: Subcortical pathways serving cortical language sites: initial experience with diffusion tensor imaging fiber tracking combined with intraoperative language mapping. Neuroimage 21:616-622, 2004

15. Hervey-Jumper SL, Li J, Lau D, Molinaro AM, Perry DW, Meng L, et al: Awake craniotomy to maximize glioma resection: methods and technical nuances over a 27 -year period. J Neurosurg 123:325-339, 2015

16. Hickok G, Poeppel D: Dorsal and ventral streams: a framework for understanding aspects of the functional anatomy of language. Cognition 92:67-99, 2004

17. Jones DK, Cercignani M: Twenty-five pitfalls in the analysis of diffusion MRI data. NMR Biomed 23:803-820, 2010

18. Keles GE, Berger MS: Advances in neurosurgical technique in the current management of brain tumors. Semin Oncol 31:659-665, 2004

19. Leemans A, Jones DK: The B-matrix must be rotated when correcting for subject motion in DTI data. Magn Reson Med 61:1336-1349, 2009

20. Makris N, Preti MG, Asami T, Pelavin P, Campbell B, Papadimitriou GM, et al: Human middle longitudinal fascicle: variations in patterns of anatomical connections. Brain Struct Funct 218:951-968, 2013
21. Mandelli ML, Berger MS, Bucci M, Berman JI, Amirbekian B, Henry RG: Quantifying accuracy and precision of diffusion MR tractography of the corticospinal tract in brain tumors. J Neurosurg 121:349-358, 2014

22. Martino J, De Witt Hamer PC, Berger MS, Lawton MT, Arnold CM, de Lucas EM, et al: Analysis of the subcomponents and cortical terminations of the perisylvian superior longitudinal fasciculus: a fiber dissection and DTI tractography study. Brain Struct Funct 218:105-121, 2013

23. Moritz-Gasser S, Herbet G, Maldonado IL, Duffau H: Lexical access speed is significantly correlated with the return to professional activities after awake surgery for low-grade gliomas. J Neurooncol 107:633-641, 2012

24. Quiñones-Hinojosa A, Ojemann SG, Sanai N, Dillon WP, Berger MS: Preoperative correlation of intraoperative cortical mapping with magnetic resonance imaging landmarks to predict localization of the Broca area. J Neurosurg 99:311-318, 2003

25. Sanai N, Martino J, Berger MS: Morbidity profile following aggressive resection of parietal lobe gliomas. J Neurosurg 116:1182-1186, 2012

26. Sanai N, Mirzadeh Z, Berger MS: Functional outcome after language mapping for glioma resection. N Engl J Med 358:18-27, 2008

27. Smith JS, Chang EF, Lamborn KR, Chang SM, Prados MD, Cha S, et al: Role of extent of resection in the long-term outcome of low-grade hemispheric gliomas. J Clin Oncol 26:1338-1345, 2008

28. Tarapore PE, Findlay AM, Lahue SC, Lee H, Honma SM, Mizuiri D, et al: Resting state magnetoencephalography functional connectivity in traumatic brain injury. J Neurosurg 118:1306-1316, 2013

29. Teixidor P, Gatignol P, Leroy M, Masuet-Aumatell C, Capelle L, Duffau H: Assessment of verbal working memory before and after surgery for low-grade glioma. J Neurooncol 81:305-313, 2007

30. Tristán-Vega A, Westin CF: Probabilistic ODF estimation from reduced HARDI data with sparse regularization. Med Image Comput Comput Assist Interv 14:182-190, 2011

31. Tuch DS: Q-ball imaging. Magn Reson Med 52:1358-1372, 2004

\section{Disclosures}

The authors report no conflict of interest concerning the materials or methods used in this study or the findings specified in this paper.

\section{Author Contributions}

Conception and design: Caverzasi, Amirbekian, Henry. Acquisition of data: Hervey-Jumper, Racine, Sankaranarayanan, Amirbekian, Papinutto, Berger, Henry. Analysis and interpretation of data: Caverzasi, Jordan, Lobach, Li, Panara, Racine, Sankaranarayanan, Berger, Henry. Drafting the article: Caverzasi, HerveyJumper, Jordan, Papinutto, Berger, Henry. Critically revising the article: Caverzasi, Hervey-Jumper, Jordan, Papinutto, Berger, Henry. Reviewed submitted version of manuscript: Caverzasi, Berger, Henry. Approved the final version of the manuscript on behalf of all authors: Caverzasi. Statistical analysis: Caverzasi, Lobach, Henry. Study supervision: Caverzasi, Berger, Henry.

\section{Correspondence}

Eduardo Caverzasi, Department of Neurology, University of California, San Francisco, Box 3206, 675 Nelson Rising Ln., Rm. 235, San Francisco, CA 94158. email: eduardo.caverzasi@ucsf. edu. 